\title{
Smooth-muscle BMAL1 participates in blood pressure circadian rhythm regulation
}

\author{
Zhongwen Xie, ${ }^{1,2}$ Wen Su, ${ }^{3}$ Shu Liu, ${ }^{3}$ Guogang Zhao, ${ }^{3}$ Karyn Esser, ${ }^{1}$ Elizabeth A. Schroder, ${ }^{1}$ Mellani Lefta, ${ }^{1}$ Harald M. Stauss, ${ }^{4}$ \\ Zhenheng Guo, ${ }^{3}$ and Ming Cui Gong ${ }^{1}$
}

'Department of Physiology, College of Medicine, University of Kentucky, Lexington, Kentucky, USA. ${ }^{2}$ Key Laboratory of Tea Biochemistry and Biotechnology, Ministry of Agriculture and Ministry of Education, Anhui Agricultural University, Anhui, China. ${ }^{3}$ Department of Internal Medicine, College of Medicine, University of Kentucky, Lexington, Kentucky, USA. ${ }^{4}$ Department of Health and Human Physiology, The University of lowa, lowa City, lowa, USA.

\begin{abstract}
As the central pacemaker, the suprachiasmatic nucleus (SCN) has long been considered the primary regulator of blood pressure circadian rhythm; however, this dogma has been challenged by the discovery that each of the clock genes present in the SCN is also expressed and functions in peripheral tissues. The involvement and contribution of these peripheral clock genes in the circadian rhythm of blood pressure remains uncertain. Here, we demonstrate that selective deletion of the circadian clock transcriptional activator aryl hydrocarbon receptor nuclear translocator-like (Bmal1) from smooth muscle, but not from cardiomyocytes, compromised blood pressure circadian rhythm and decreased blood pressure without affecting SCN-controlled locomotor activity in murine models. In mesenteric arteries, BMAL1 bound to the promoter of and activated the transcription of Rho-kinase 2 (Rock2), and Bmal1 deletion abolished the time-of-day variations in response to agonist-induced vasoconstriction, myosin phosphorylation, and ROCK2 activation. Together, these data indicate that peripheral inputs contribute to the daily control of vasoconstriction and blood pressure and suggest that clock gene expression outside of the SCN should be further evaluated to elucidate pathogenic mechanisms of diseases involving blood pressure circadian rhythm disruption.
\end{abstract}

\section{Introduction}

Human blood pressure undergoes daily oscillations: blood pressure is lowest at night (nocturnal dip) and rises before awakening (morning surge) (1). Such blood pressure circadian rhythm is critical to human health, as a $40 \%$ higher risk of acute myocardial infarction, a $29 \%$ higher risk of sudden cardiac death, and a $49 \%$ higher risk of stroke occur during the early morning blood pressure surge compared with the rest of the day $(2,3)$. It was long believed that blood pressure circadian rhythm, just like other physiological and behavioral circadian rhythms, was mostly controlled by the suprachiasmatic nucleus (SCN). However, this dogma was challenged by the discoveries that each of the core clock genes that exists in the SCN also expresses and functions in peripheral tissues (4-7). These discoveries raise some fundamental questions: do these peripheral clock genes participate in the regulation of blood pressure circadian rhythm? And if so, which specific clock genes in which peripheral tissue or tissues are important for blood pressure circadian rhythm?

Smooth muscle is a major component of the blood vessel wall, and its primary physiological function is to maintain adequate organ blood supply and blood pressure homeostasis by adjusting its contractile state in response to environmental cues (8). Smoothmuscle contractility exhibits time-of-day variation (9-14). However, whether and how such time-of-day smooth-muscle contractile

Authorship note: Zhongwen Xie and Wen Su contributed equally to this work. Conflict of interest: The authors have declared that no conflict of interest exists. Submitted: May 2, 2014; Accepted: November 6, 2014.

Reference information: J Clin Invest. 2015;125(1):324-336. doi:10.1172/JCI76881. variation relates to blood pressure circadian rhythm are unknown. Moreover, the molecular mechanism that underlies time-of-day smooth-muscle contractile variation is unknown.

Aryl hydrocarbon receptor nuclear translocator-like (BMAL1), also known as Arntl3 in mouse and MOP3 in humans, is a central element of the core clock genes in mammals. Global Bmal1 deletion in mice causes immediate and complete loss of rhythmicity, including in blood pressure circadian rhythm $(15,16)$. BMAL1 is expressed ubiquitously but functions in a tissue-specific manner. For instance, it has been shown that brain-specific BMAL1 expression in global Bmal1-knockout mice selectively restored wheel-running circadian rhythm, and skeletal muscle-specific BMAL1 expression selectively restored wheel-running level and body weight (17). However, the physiological function of BMAL1 in smooth muscle and its contribution to blood pressure circadian rhythm remain to be defined. The current study developed a smooth-muscle-specific Bmal1-knockout mouse model and demonstrated in vitro and in vivo that smooth-muscle BMAL1 is essential for normal vascular smooth-muscle contraction amplitude and time-of-day variations as well as blood pressure level and circadian rhythm.

\section{Results}

Generation and characterization of a smooth-muscle-specific Bmal1knockout mouse model. We generated a smooth-muscle-specific Bmal1-knockout mouse model (SM-Bmal1-KO) by crossing

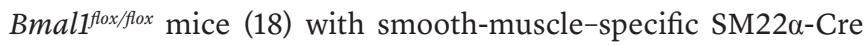
mice (19). SM-Bmal1-KO mice were viable, fertile, and grossly normal. Four distinct approaches were taken to characterize 
SM-Bmal1-KO mice. First, SM-Bmal1-KO mice exhibited a Cremediated chromosome recombination that specifically deletes the Bmal1 gene in smooth-muscle-enriched tissues, such as the aorta, mesenteric artery, and bladder (Figure 1A). Second, Bmal1 mRNA (Figure 1B) and protein (Figure 1C) were drastically decreased in mesenteric arteries from the SM-Bmal1-KO mice compared with those in WT littermates (Bmalf flox/flox mice). Third, the 24-hour mRNA oscillations of period circadian clock 1 (Per1) and period circadian clock 2 (Per2), 2 BMAL1 target genes, were abolished in mesenteric arteries by Bmal1 deletion (Figure 1, D and $\mathrm{E})$. In addition, in agreement with the literature $(19,20)$, we also found that Cre recombinase was indeed expressed in heart but at a much lower level than in smooth muscle (Z. Guo and M. Gong, unpublished observations). In contrast to the drastic effect of Bmal1 deletion in the mesenteric artery, Bmal1 deletion in the heart had no effect on Per1 and Per2 mRNA 24-hour oscillation (Figure 1, F and G). Finally, PER2 oscillation was monitored in real time by measuring luminescence in mesenteric arteries or SCNcontaining brain slices isolated from SM-Bmal1-KO/mPer2 $2^{\mathrm{Luc}}$ knockin mice. PER2 oscillations were completely lost in mesenteric arteries (Figure 1H), but remained normal in SCN-containing brain slices (Figure 1I).

In addition, we measured the body weight, body fat mass, fasting blood glucose, glucose tolerance, and insulin tolerance in SM-Bmal1-KO mice and WT littermates to determine whether the disruptions of adipogenesis and glucose metabolism reported in global Bmal1-KO mice (21) are present in the SM-Bmal1-KO mice. No difference was detected in any of these indices (Z. Guo and M. Gong, unpublished observations). We also measured kidney weight and length to determine whether the reduction of kidney mass in the absence of degenerative lesions reported in global Bmal1-KO mice (22) occurs in SM-Bmal1-KO mice. No difference was found between SM-Bmal1-KO mice and WT littermates (Z. Guo and M. Gong, unpublished observations). Consistent with those findings, we also did not find any significant difference in urine volume and blood sodium concentration between SM-Bmal1-KO mice and WT littermates (Z. Guo and M. Gong, unpublished observations).

Time-of-day variations in isolated vascular smooth-muscle contraction and in vivo pressor responses are suppressed in SM-Bmal1-KO mice. To investigate the role of smooth-muscle BMAL1 in vascular tone, superior mesenteric arteries were isolated from SM-Bmal1-KO mice and WT littermates at zeitgeber time 5 (ZT5) and ZT17. Endothelium was denuded, and their contractile responses to high potassium $\left(\mathrm{K}^{+}\right)$, phenylephrine $(\mathrm{PE})$, and serotonin $(5-\mathrm{HT})$ were determined. Consistent with our recent report in abdominal aorta (9), the contractile responses to all 3 stimuli in mesenteric arteries were higher at ZT5 than at ZT17 in WT littermates (Figure 2, A-E). Strikingly, smoothmuscle-specific Bmal1 deletion abolished the time-of-day variations in smooth-muscle contractile responses to $\mathrm{K}^{+}$(Figure 2A), PE (Figure 2, B and C), and 5-HT (Figure 2, D and E). In addition, the maximal contractile responses to all 3 stimuli were markedly suppressed at both ZT5 and ZT17 in SM-Bmal1-KO mice compared with WT littermates (Figure 2, A, C, and E).

We next examined the effect of smooth-muscle BMAL1 on vascular structure. We did not find significant differences in the medium thickness, medium area, lumen area, and ratio of medium to lumen in the second branch of mesenteric arteries between SM-Bmal1-KO mice and WT littermates (Supplemental Figure 1, A and B; supplemental material available online with this article; doi:10.1172/ JCI76881DS1). These data suggest that smooth-muscle Bmal1 deletion has little effect on vascular structure and, thus, that the suppression of contraction amplitude in tissues from SM-Bmal1-KO mice is unlikely to be attributed to vascular structure changes.

We also determined the contractile responses to $\mathrm{K}^{+}, \mathrm{PE}$, and 5 -HT in right renal arteries and found suppression in contractile time-of-day variation and amplitude similar to that found in mesenteric arteries in SM-Bmal1-KO mice (Supplemental Figure 2, $\mathrm{A}-\mathrm{E})$. These data suggest that the effect of smooth-muscle BMAL1 on contractile responses is not limited to mesenteric arteries.

In addition to responding to neuronal and humoral stimulations, vascular smooth muscle in resistance arterioles contracts in response to mechanical stretch via a mechanism termed myogenic responses. To determine the role of smooth-muscle BMAL1 in myogenic responses in small resistance arteries that are more relevant to blood pressure, we investigated the myogenic responses in the fifth branch of mesenteric arteries (lumen diameters of 55 to $85 \mu \mathrm{m}$ ) isolated from SM-Bmal1-KO mice and WT littermates at ZT5, a time point when a more pronounced difference in $\mathrm{K}^{+}-$, PE-, and 5-HTinduced contraction was detected between the 2 mouse strains (Figure 2, A-E). As shown in Figure 3, A and B, in the presence of extracellular $\mathrm{Ca}^{2+}$, when the vascular smooth muscle can generate active myogenic contraction, the vessel lumen diameter was increased to a larger extent in response to the pressure steps from 20 to 120 $\mathrm{mmHg}$ in SM-Bmal1-KO mice than in WT control mice, indicating a compromised myogenic response in SM-Bmal1-KO mice. Consistent with this result, when the average spontaneous tone at the physiological pressure of $60 \mathrm{mmHg}$ was calculated as the percentage decrease in active lumen diameter from the passive diameter (23), vessels from SM-Bmal1-KO mice exhibited significantly less spontaneous tone than those in WT littermates (Figure 3B).

When the active myogenic response was eliminated in the absence of added $\mathrm{Ca}^{2+}$ and the presence of EGTA plus nitroprusside, the difference in vessel lumen increase between 2 strains of mice was largely diminished (Figure 3A; passive vs. active), indicating that a compromised smooth-muscle myogenic response rather than a vascular structure change is largely responsible for the difference between SM-Bmal1-KO mice and WT controls. Nevertheless, it was noted that a small but significant $(P<0.05)$ difference remained in the absence of $\mathrm{Ca}^{2+}$ between the 2 strains of mice, suggesting that there is a moderate structural change in the fifth branch of mesenteric arteries from SM-Bmal1-KO mice. Consistent with this interpretation, there were trends toward a decrease in the cross-sectional lumen area and an increase in the cross-sectional wall area and, as a result, a small but significant increase in wall-to-lumen ratio (Supplemental Figure 3, A-D).

To further verify whether the vascular contractile differences detected in isolated vascular preparations are present in vivo, we determined the immediate pressor responses to i.v. injection of various doses of PE at ZT5 and ZT17. Similar to what we observed in isolated mesenteric arteries (Figure 2) and renal arteries (Supplemental Figure 2) from WT littermates, the pressor responses to 10 or $50 \mu \mathrm{g} / \mathrm{kg}$ PE at ZT5 were significantly higher than those at ZT17, whereas such time-of-day difference was abolished in 
A

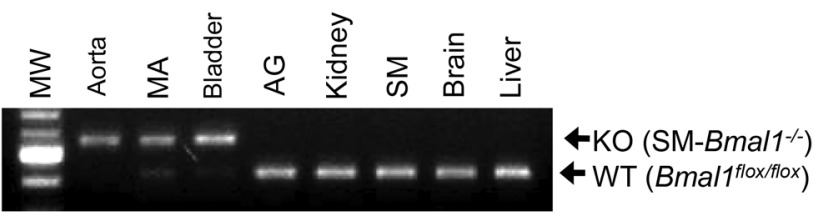

B

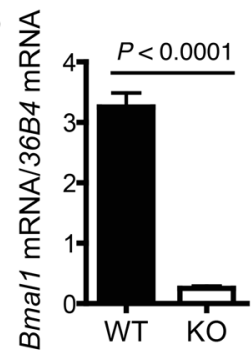

C

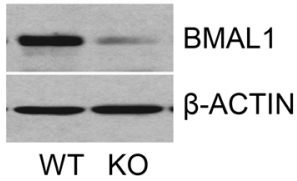

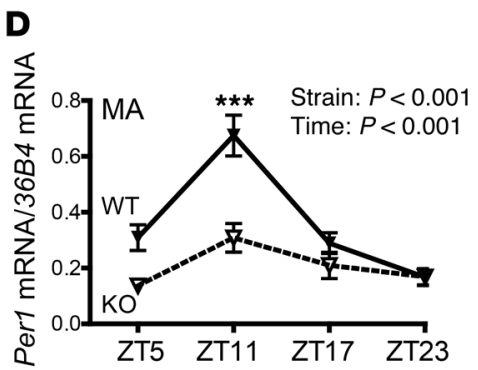

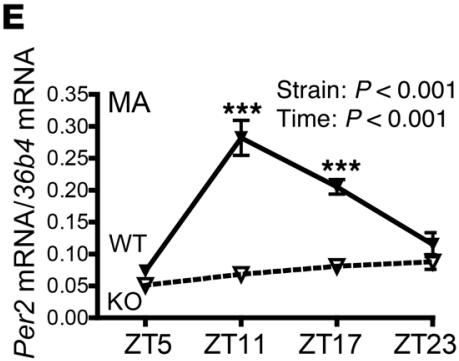

H
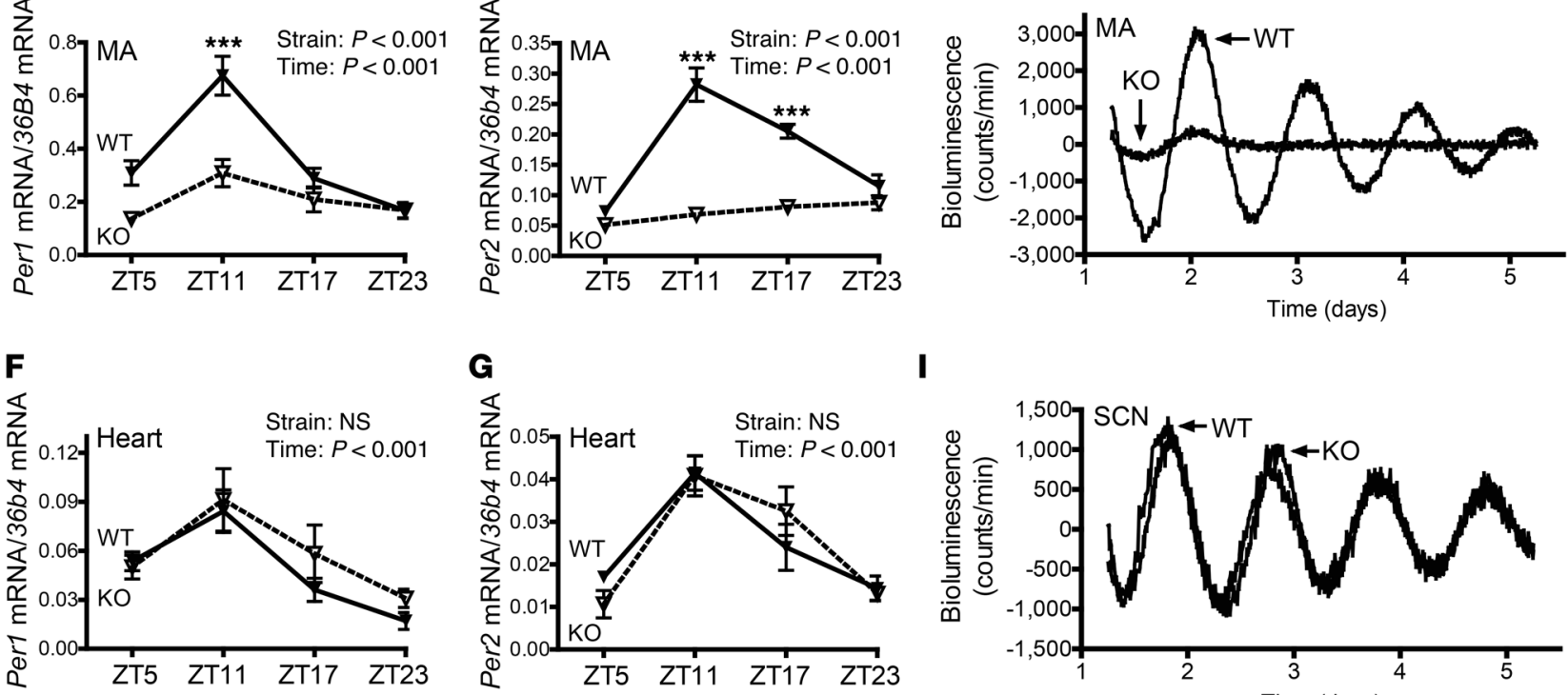

I

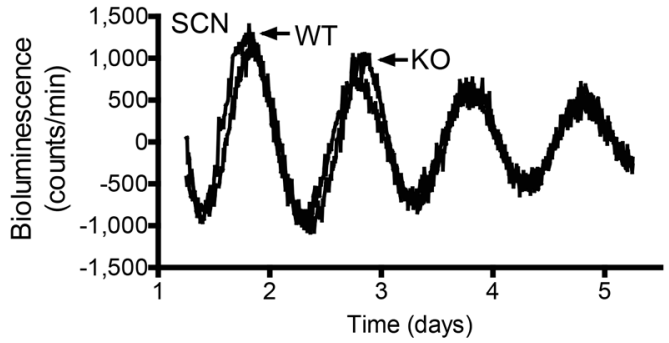

Figure 1. Characterization of SM-Bmal1-KO mice. (A) Analysis of smooth-muscle-specific Cre-mediated chromosome recombination by PCR using genomic DNA from SM-Bmal1-KO mice. MA, mesenteric artery; AG, adrenal gland; SM, skeletal muscle. (B) Real-time PCR analysis of Bmal1 mRNA expression in mesenteric arteries $(n=3-5)$. (C) Representative Western blots of BMAL1 and $\beta$-actin protein expression in mesenteric arteries $(n=8)$. (D-C) Real-time PCR analysis of Per1 ( $\mathbf{D}$ and $\mathbf{F}$ ) and Per2 (E and $\mathbf{G})$ mRNA expression in mesenteric arteries ( $\mathbf{D}$ and $\mathbf{E}$ ) and the heart ( $\mathbf{F}$ and $\mathbf{G})(n=3-5)$. ${ }^{* * *} P<0.001$ vs. KO at ZT11. ( $\mathbf{H}$ and $\mathbf{I})$ Representative LumiCycle luminescence recordings of PER2-luciferase protein oscillation in MA (H) and SCN-containing brain slice (I).

SM-Bmal1-KO mice (Figure 3C and Supplemental Table 1). In addition, the pressor responses to 10 or $50 \mu \mathrm{g} / \mathrm{kg}$ PE at ZT5 were smaller in SM-Bmal1-KO mice compared with those in WT littermates (Figure 3C and Supplemental Table 1).

Taken together, these data demonstrate that smooth-muscle BMAL1 is essential for the 24-hour variations and normal amplitude of contractile responses in vitro and in vivo.

$M L C_{20}$ phosphorylation increase is selectively inhibited at ZT5 but not ZT17 in mesenteric arteries from SM-Bmal1-KO mice. Reversible phosphorylation of the 20-kDa regulatory myosin light chain $\left(\mathrm{MLC}_{20}\right)$ is a primary mechanism regulating smooth-muscle contraction (8); we therefore investigated the possibility that smooth-muscle BMAL1 may mediate the time-of-day variation of contraction through regulating $\mathrm{MLC}_{20}$ phosphorylation. Mesenteric arteries were isolated from SM-Bmal1-KO mice and WT littermates at ZT5 or ZT17 and then incubated with PE and 5-HT to induce $\mathrm{MLC}_{20}$ phosphorylation. We used both $\mathrm{PE}$ and 5-HT rather than a single agonist to stimulate $\mathrm{MLC}_{20}$ phosphorylation to activate various branches of mesenteric arteries, as the contraction amplitude induced by PE or 5-HT varied at the different branches of mesenteric arteries (ref. 24 and Figure 2, B-E). Moreover, 2 agonists were expected to drive the $\mathrm{MLC}_{20}$ phosphorylation to a higher level, thus providing a relatively larger window for the detection of a decrease in $\mathrm{MLC}_{20}$ phosphorylation in SM-Bmal1-KO mice. Two distinct methods, urea glycerol gel electrophoresis that separates un-, mono-, and diphosphorylated $\mathrm{MLC}_{20}(24)$ and Western blot using antibodies specific for phosphorylated $\mathrm{MLC}_{20}$ (Thr18/Ser19), were used to determine MLC $_{20}$ phosphorylation.

In WT littermates, stimulation of the vessels with PE plus 5-HT significantly increased $\mathrm{MLC}_{20}$ phosphorylation at both ZT5 (Figure 4, A-D, lanes 1 vs. 2) and ZT17 (Figure 4, A-D, lanes 5 vs. 6), but the amplitude of $\mathrm{MLC}_{20}$ phosphorylation increase at ZT5 was significantly higher than that at ZT17 (Figure 4, A-D, lanes 2 vs. 6). These results correlate with the larger contractile responses at ZT5 relative to at ZT17 (Figure 2 and Supplemental Figure 2) and suggest that the time-of-day variation in contractile amplitude is mediated, at least in part, by $\mathrm{MLC}_{20}$ phosphorylation.

In SM-Bmal1-KO mice, stimulation of the vessels with PE plus 5-HT also increased MLC $_{20}$ phosphorylation at both ZT5 (Figure 4, A-D, lanes 3 vs. 4) and ZT17 (Figure 4, A-D, lanes 7 vs. 8). However, the amplitude of $\mathrm{MLC}_{20}$ phosphorylation increase in SM-Bmal1KO mice was selectively suppressed at ZT5 (Figure 4, A-D, lanes 2 
A

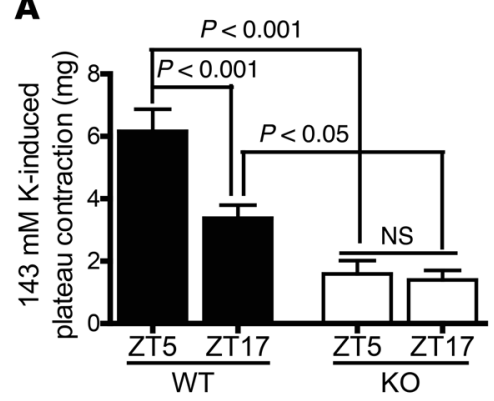

B

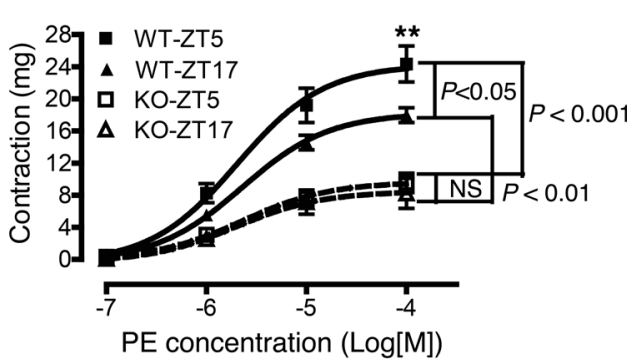

C

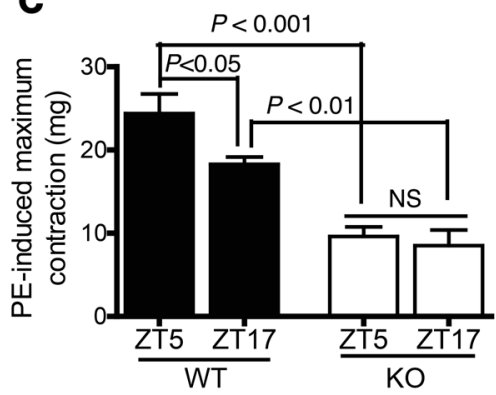

D

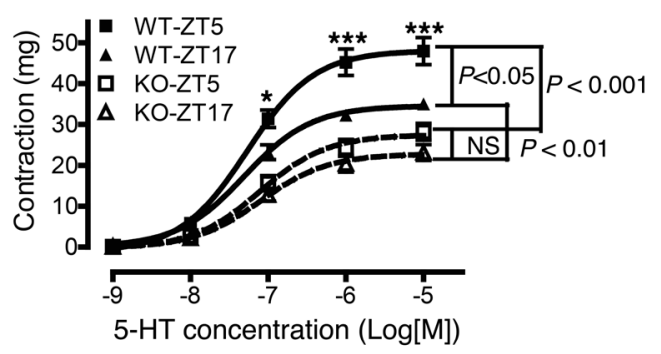

E

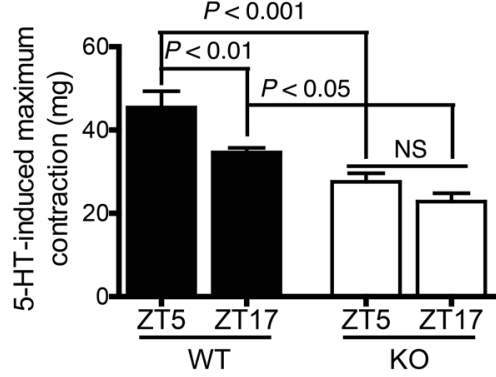

Figure 2. The time-of-day variation in agonist-induced contractile responses is suppressed in superior mesenteric artery from SM-Bmal1-KO mice. (A) The plateau response to $143 \mathrm{mM} \mathrm{K}^{+}(n=4-8)$. (B and $\left.\mathbf{C}\right)$ The concentration-response curve $(\mathbf{B})$ and the maximum response $(\mathbf{C})$ to $P E(n=6-9)$. ${ }^{*} P<0.01$ vs. WT-ZT17 at $100 \mu \mathrm{M}$ PE (D and E). The concentration-response curve (D) and the maximum response $(\mathbf{E})$ to $5-\mathrm{HT}(n=6-9)$. ${ }^{*} P<0.05$ vs. WT-ZT17 at $0.1 \mu \mathrm{M}$ $5-\mathrm{HT} ;{ }^{* *} \mathrm{P}<0.001$ vs WT-ZT17 at 1 or $10 \mu \mathrm{M}$ 5-HT.

vs. 4), but not at ZT17 (Figure 4, A-D, lanes 6 vs. 8), compared with that in WT littermates. As a consequence, the difference in the $\mathrm{MLC}_{20}$ phosphorylation between ZT5 and ZT17 was abolished in SM-Bmal1-KO mice, which correlates with the abolishment of the differences in contractile responses between ZT5 and ZT17 in SMBmal1-KO mice (Figure 2 and Supplemental Figure 2).

Identification of ROCK 2 as a new target of BMAL1 in mesenteric arteries. To identify molecular mechanisms that underlie agonistinduced and BMAL1-mediated smooth-muscle contraction, we investigated several proteins that are either essential components of contractile apparatus or key regulators of $\mathrm{MLC}_{20}$ phosphorylation. By immunoblot analysis, we compared the expression levels of the following proteins at ZT5 in mesenteric arteries from SMBmal1-KO and WT littermates: (a) $\alpha$-SMA; (b) $\mathrm{MLC}_{20}$; (c) myosin phosphatase target subunit 1 (MYPT1); (d) 17-kDa PKC-potentiated protein phosphatase-1 inhibitor (CPI-17); (e) Rho-kinase 2 (ROCK2); and (f) small-molecular weight G protein RhoA. Interestingly, among all the proteins investigated, we found that ROCK2 was selectively decreased in SM-Bmal1-KO mice compared with that in WT littermates (Supplemental Figure 4, A-G). Since ROCK2 is a key regulator of smooth-muscle contraction and blood pressure homeostasis and its dysfunction has been implicated in many cardiovascular diseases (25), we therefore tested the hypothesis that ROCK2 links BMAL1 and time-of-day variations in vascular smooth-muscle contraction by various approaches.

First, to determine whether BMAL1, as a transcriptional factor, directly binds Rock2 promoter, we analyzed the mouse Rock2 promoter DNA sequence and identified multiple canonical E-boxes (CANNTG, where N can be any nucleotide) that BMAL1 can poten- tially bind to (Figure 5A). To determine whether BMAL1 binds to these putative E-boxes, we generated a rabbit polyclonal antibody that specifically recognized BMAL1 (Figure 5B) and performed a ChIP assay in mesenteric arteries isolated from WT mice at ZT5 and ZT17. As a positive control, we examined whether BMAL1 binds to the Per1 (a classic BMAL1 target) promoter and found that indeed it did, as expected (Figure 5C). Importantly, we found that BMAL1 bound to the Rock2 promoter at E-box 5 through E-box 8, but not at E-box 9 and E-box 10 (Figure 5, A and C). Moreover, more BMAL1 binding was detected at ZT17 than at ZT5, indicating that BMAL1 binds to the Rock2 promoter in a time-of-day-dependent manner (Figure 5, C and D).

Second, to investigate whether the binding of BMAL1 to the Rock2 promoter regulates its activity, we cloned a 3.2-kb mouse Rock2 promoter, inserted it into a luciferase reporter vector (pGl3Rock2P-Luc), and transfected the pGl3-Rock2P-Luc vector into aortic vascular smooth-muscle cells (VSMCs) isolated from SMBmal1-KO mice and WT littermates. In WT cells, the Rock2 promoter exhibited a 14-fold increase in luciferase activity over the pGL3 luciferase vector (Figure 5E, column 1 vs. 2). In contrast, when transfected into Bmal1-deficient cells, Rock2 promoter activity was abolished (Figure 5E, column 2 vs. 4), suggesting that BMAL1 is required for Rock2 promoter activity in cultured VSMCs. Importantly, infection of Bmal1-deficient cells by BMAL1 adenovirus almost completely restored Rock2 promoter activity in a concentration-dependent manner (Figure 5E, column 5 and 6 vs. 4).

Third, we investigated whether smooth-muscle Bmal1 deletion affects Rock2 mRNA expression in vivo. Mesenteric arteries were isolated from SM-Bmal1-KO and WT littermates at ZT5 and 
A

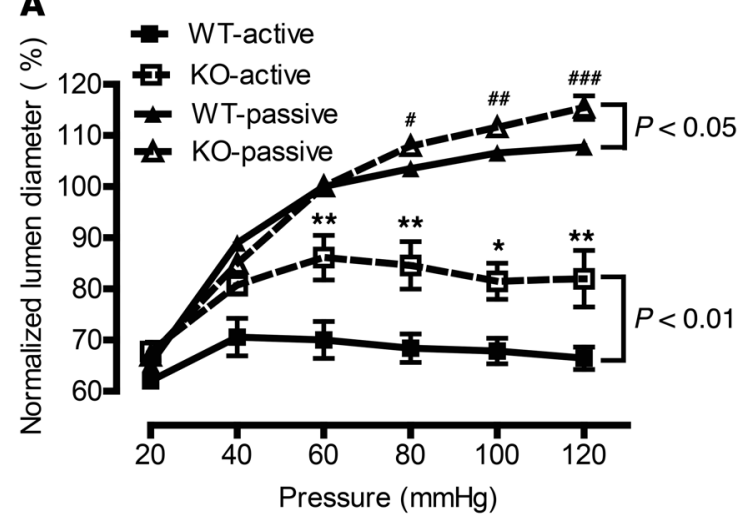

\section{C}

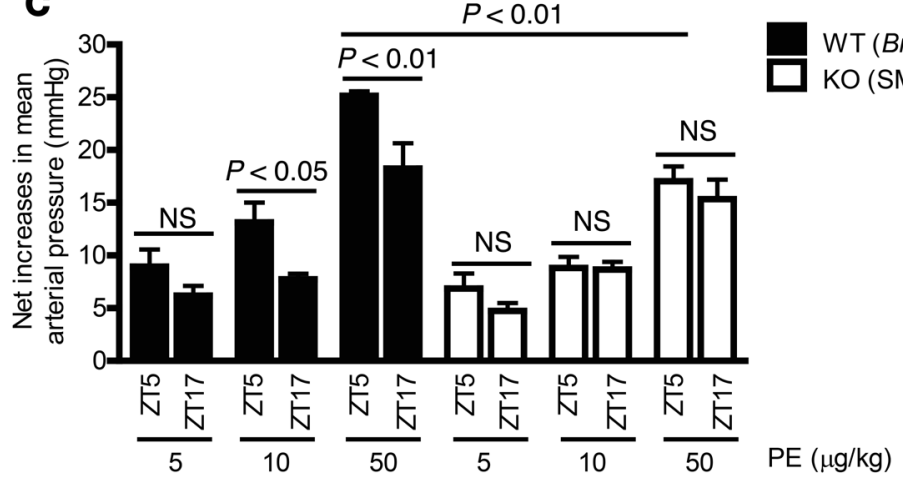

B

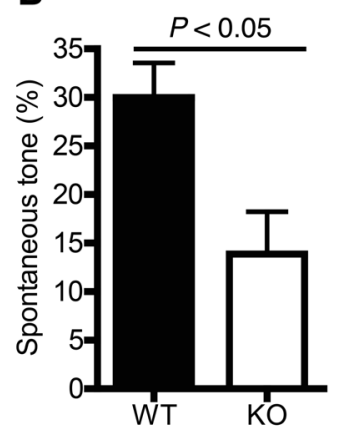

MYPT1 phosphorylation between ZT5 and ZT17 was abolished in SM-Bmal1-KO mice.

Finally, we investigated whether pharmacological inhibition of ROCK2 suppresses vascular smooth-muscle contraction in a manner similar to that of genetic-deleting Bmal1. Endothelium-denuded superior mesenteric arteries were prepared from WT mice at ZT5 and ZT17 and then stimulated with 5-HT in the presence of Rho kinase inhibitor Y-27632 or vehicle (DMSO). The results demonstrate that $\mathrm{Y}-27632$ suppressed 5-HTinduced contractions at both ZT5 and ZT17 (Figure 6D). Importantly, the extent of suppression on the contraction was larger at ZT5 than at ZT17; thus, the difference in 5-HTinduced contraction between ZT5 and ZT17 was abolished by Rho kinase blockade, which is similar to that in SM-Bmal1-KO mice (Figure 2 and Supplemental Figure 2).

Impairment of blood pressure circadian rhythm in SM-Bmal1-KO mice. To investigate whether smooth-muscle BMAL1 is involved in blood pressure circadian rhythm, we measured blood pressure of conscious, free-moving SM-Bmal1-KO mice and WT littermates using telemetry under 12:12 light/dark (L/D), constant dark (D/D), and constant light (L/L) conditions. Mean arterial pressure (MAP) was significantly decreased under all 3 conditions in SM-Bmal1-KO mice compared with that in WT littermates (Figure 7A).

To investigate whether smooth-muscle BMAL1 is involved in the intrinsic blood pressure circadian rhythm, we first determined

ZT17, and Rock2 mRNA was quantified by real-time PCR. In WT vessels, Rock2 mRNA was significantly higher at ZT17 than that at ZT5 (Figure 6A). In BMAL1-deficient vessels, Rock2 mRNA expression was diminished at both ZT5 and ZT17, but the decrease was more dramatic at ZT17 than at ZT5 (Figure 6A). As a result, there was no difference in Rock2 mRNA between ZT5 and ZT17 in Bmal1-deficient mesenteric arteries.

Fourth, we investigated whether BMAL1 regulates the timeof-day variations in ROCK2 activity. We determined the phosphorylation level of MYPT1 at Thr853 (an index of ROCK2 activation) in mesenteric arteries isolated from SM-Bmal1-KO mice and WT littermates at ZT5 and ZT17. In WT vessels, PE plus 5-HT significantly increased MYPT1 phosphorylation at ZT5 (Figure 6, B and C, lane 1 vs. 2) and ZT17 (Figure 6, B and C, lane 5 vs. 6). The amplitude of agonist-induced MYPT1 phosphorylation at ZT5 was significantly higher than that at ZT17 (Figure 6, B and C, lane 2 vs. 6), which is consistent with larger contractile responses (Figure 2 and Supplemental Figure 2) and $\mathrm{MLC}_{20}$ phosphorylation (Figure 4) at ZT5 than at ZT17 in WT mice. In contrast, in SM-Bmal1-KO mice, agonist-induced MYPT1 phosphorylation was selectively reduced at ZT5 (Figure 6, B and C, lane 2 vs. 4), but not ZT17 (Figure $6, \mathrm{~B}$ and $\mathrm{C}$, lane 6 vs. 8). Consequently, the difference in the blood pressure in the absence of the dominant external light cue under D/D conditions (26). Systolic blood pressure (SBP), diastolic blood pressure (DBP), and pulse pressure were continuously measured for 7 consecutive days. As shown in Figure 7B, SMBmal1-KO mice exhibited a decrease in SBP during the subjective dark phase without an apparent change during the subjective light phase. An analysis of the data using a nonlinear least-square fitting program, PHARMFIT (27), illustrated that the SBP circadian oscillation amplitude was significantly decreased (Figure 7C) and the acrophase (time of the peak) was significantly shifted forward (Figure 7D), but the period length (the time elapsed for 1 complete oscillation) was unaltered (Figure 7E) in SM-Bmal1-KO mice relative to WT littermates.

A similar but more dramatic effect of smooth-muscle BMAL1 on blood pressure circadian rhythm was also observed in DBP (Figure 7F). The pressure levels during both the subjective dark and light phases were decreased in the SM-Bmal1-KO mice, but the amplitude of decrease was bigger during the subjective dark phase than during the subjective light phase. Consequently, the amplitude of DBP oscillation was substantially decreased. The acrophase of DBP also shifted forward. Similar to SBP, the period length of the DBP was not different between the 2 strains of mouse. 
A

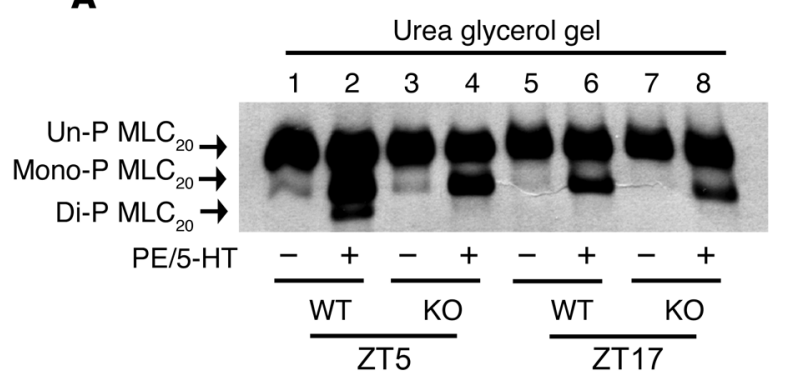

C

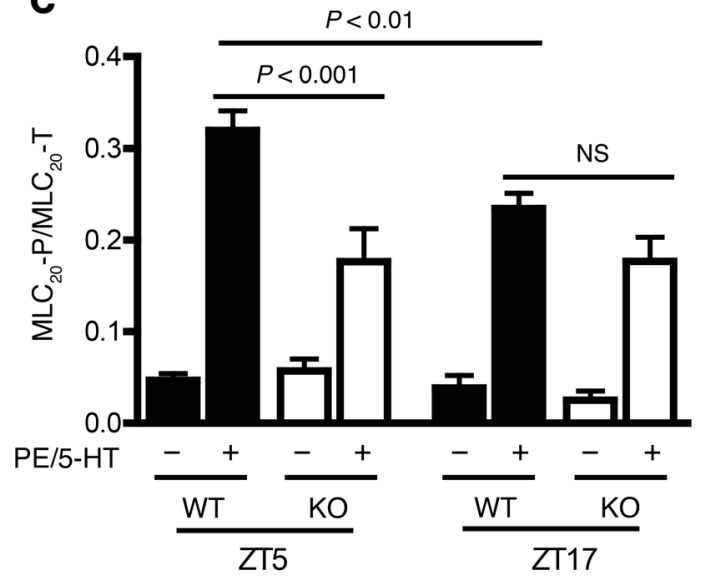

B

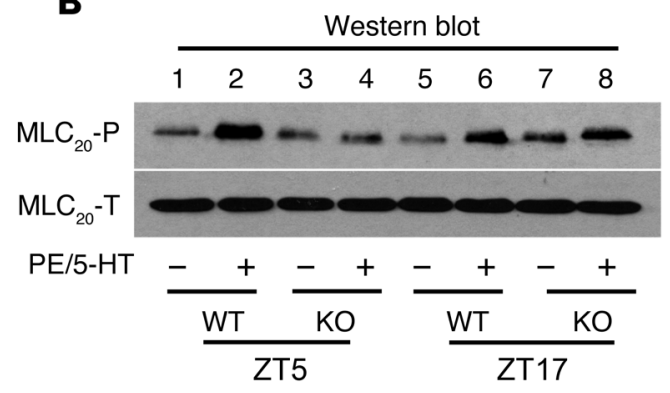

D

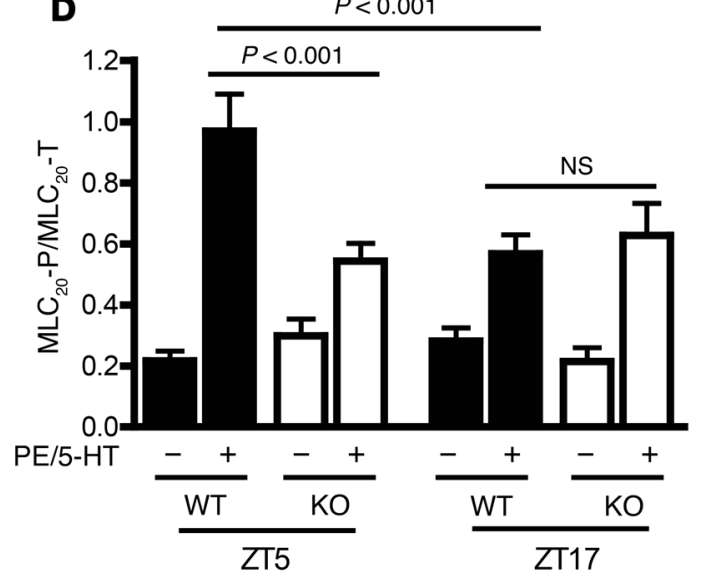

Figure 4. The time-of-day variations in agonist-induced $\mathrm{MLC}_{20}$ phosphorylation are diminished in mesenteric arteries from SM-Bmal1-KO mice. (A and C) Representative urea/glycerol gel (A) and quantitative data ( $\mathbf{C} ; n=7)$ of $\mathrm{MLC}_{20}$ phosphorylation induced by PE (100 $\left.\mu \mathrm{M}\right)$ plus 5 -HT (10 $\left.\mu \mathrm{M}\right)$ for 2 minutes.

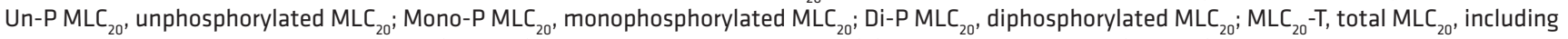

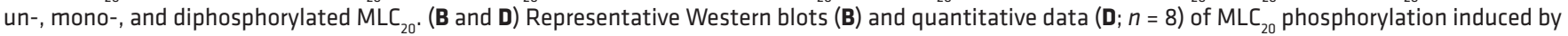
PE plus 5-HT using the antibodies specific for phosphorylated $\mathrm{MLC}_{20}$ (Thr18 and Ser19) and total $\mathrm{MLC}_{20}$ protein.

The most striking effect of smooth-muscle BMAL1 on blood pressure circadian rhythm was seen in pulse pressure (Figure $7 \mathrm{G}$ ). The level was significantly higher in the SM-Bmal1-KO mice than in the WT littermates due to a larger decrease in DBP than in SBP. In addition, JTK-cycle analysis (28) of the pulse pressure illustrated that the circadian oscillation of pulse pressure observed in WT littermates was abolished in SM-Bmal1-KO mice.

Neither the level nor the circadian oscillations of the locomotor activity, however, were altered in the SM-Bmal1-KO mice compared with WT littermates (Figure 7H). This result is consistent with the data showing that the Bmall gene remains intact in brain (Figure 1A) and that its target protein PER2 oscillation is normal (Figure 1I), indicating that the suppressed amplitude and shifted acrophase of blood pressure circadian oscillation in SM-Bmal1-KO mice are not due to changes in the SCN central pacemaker.

We also examined the effect of smooth-muscle BMAL1 on the heart rate (HR). Interestingly, smooth-muscle Bmal1 deletion decreased the HR during both the subjective dark and light phases (Figure 7I), but had no effect on the HR circadian oscillation amplitude (Figure 7J), acrophase (Figure 7K), and period length (Figure 7L). These results are consistent with the data showing that Per1 and Per 2 mRNA oscillation remains normal in the heart (Figure 1, F and G), indicating that the suppressed amplitude and shifted acrophase of blood pressure circadian oscillation in SMBmal1-KO mice are unlikely to be attributed to changes in the HR.
To investigate whether the effect of smooth-muscle Bmal1 deletion on blood pressure circadian rhythm is exacerbated by light, we then determined blood pressure for 7 consecutive days in the presence of $\mathrm{L} / \mathrm{L}$ when the SCN central pacemaker was disrupted (26). We found Bmal1 deletion in smooth muscle caused changes in BP (Supplemental Figure 5) very similar to those that occurred under D/D conditions, including a decrease in SBP, DBP, MAP, and HR (Supplemental Figure 5, A, E, F, and I), a decrease in the amplitude of SBP oscillation (Figure 7B) and no change in the acrophase and the period length of SBP oscillations (Figure 7, C and D), an increase in pulse pressure (Supplemental Figure 5G), and no effect on locomotor activity and HR circadian rhythm (Supplemental Figure 5, H-L).

Interestingly, there were 2 changes in blood pressure circadian rhythm observed in both SM-Bmal1-KO mice and WT littermates under $\mathrm{L} / \mathrm{L}$ conditions when compared with those under D/D conditions. First, under L/L conditions, only 1 single peak was observed in SBP, DBP, locomotor activity, and HR during the subjective dark phase (Supplemental Figure 5, A, E, F, H, and I), whereas under D/D conditions, 2 peaks were observed during the subjective dark phase: one at the beginning and another at the end of the subjective dark phase (Figure 7, B, F, H, and I). Second, the period length of SBP oscillation was longer: $25.0 \pm$ $0.1(\mathrm{~L} / \mathrm{L})$ vs. $24.0 \pm 0.03(\mathrm{D} / \mathrm{D})$ hours $(n=12$ each, $P<0.0001)$. The peak of the SBP, DBP, and MAP as well as locomotor activity 
A

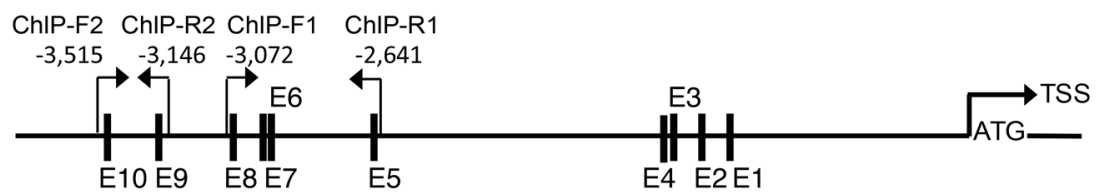

B

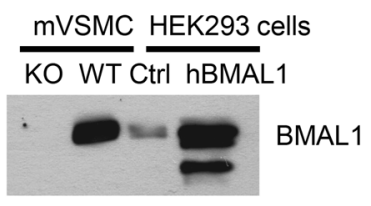

C

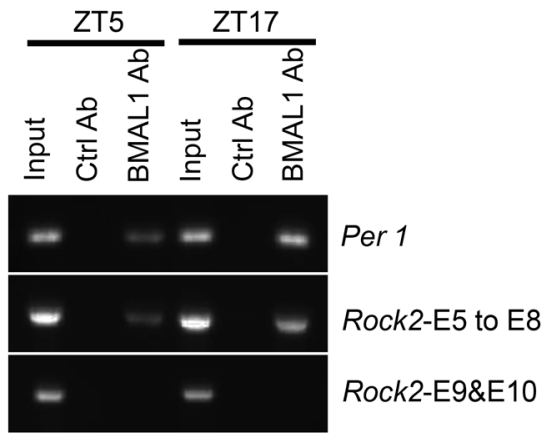

D

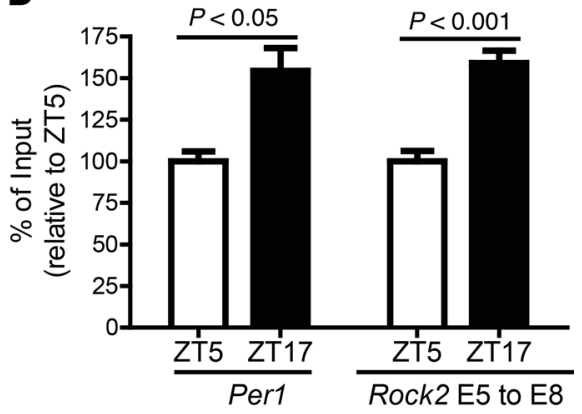

$\mathbf{E}$

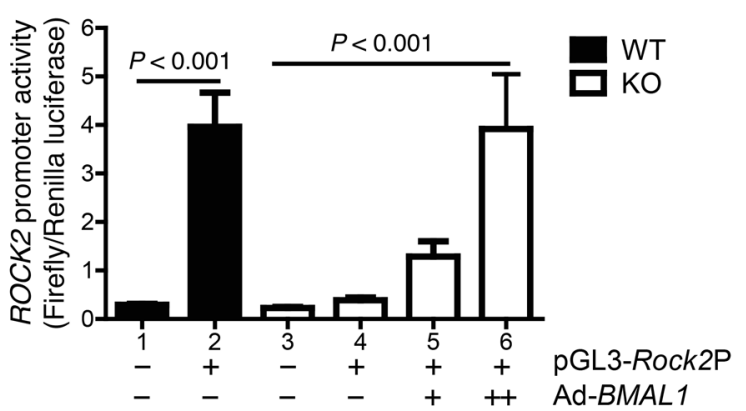

Figure 5. BMAL1 binds to Rock2 promoter in mesenteric arteries and is required for Rock2 promoter activity in cultured VSMCs. (A) Schematic diagram of a 3.5-kb mouse Rock2 promoter showing the positions of putative E-boxes (E) 1 to 10 and ChIP PCR primers relative to the translational start site (TSS). ChIP-F, forward primers; ChIP-R, reverse primers. (B) Characterization of the BMAL1 antibody by Western blots using cell lysate from Bmal1-deficient (KO) and WT mouse aortic VSMCs (mVSMC) as well as human embryonic kidney 293 cells (HEK293), transfected with a pSport6 control vector (Ctrl) or a pSport6 vector expressing human BMAL1 (hBMAL1). (C and D) Representative ChIP-PCR (C) and quantitative data $(\mathbf{D} ; n=4)$ show that BMAL1 binds to the Per1 promoter and the Rock2 promoter at E-boxes 5 to 8, but not E-boxes 9 to 10, in a time-of-day-dependent manner. (E) Restoration of Rock2 promoter activity by BMAL1 adenovirus-mediated gene expression in Bmal1-deficient mVSMCs (+, low dose; ++, high dose; $n=3-6$ ).

A moderate Bmal1 deletion was seen in the heart in SM-Bmal1-KO mice (Z. Guo and M. Gong, unpublished observations), which is consistent with a moderate decrease in the HR in SM-Bmal1-KO mice (Figure 7I, Supplemental Figure 5I, and Supplemental Figure 6I). To ensure that the observed blood pressure alterations in SM-Bmal1KO mice resulted from smooth-muscle Bmal1 deletion but not cardiomyocyte Bmal1 deletion, we measured blood pressure in an inducible cardiomyocytespecific Bmal1-knockout mouse model (iCS-Bmal1$\mathrm{KO}$ ), which was generated

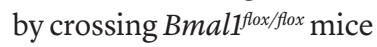
(18) with cardiac-specific $\mathrm{MerCreMer}$ recombinase mice (29), as we previously reported (30). As shown in Supplemental Figure 7B, under 12:12 L/D conditions, neither the MAP level nor the circadian oscillations of the MAP were altered in the iCS-Bmal1-KO mice compared with those in iCSBmal1-WT mice.

To test the possibility that the loss of smooth-

and HR during the subjective dark phase shifted gradually from in the middle of the subjective dark phase to the end of the subjective dark phase over the 7-day period (Supplemental Figure 5, A, E, F, H, and I).

We also determined the blood pressure oscillation under 12:12 $\mathrm{L} / \mathrm{D}$ conditions to investigate whether the external light cue was able to correct the compromised blood pressure circadian oscillation in the SM-Bmal1-KO mice. As shown in Supplemental Figure 6, A-L, changes observed in SBP, DBP, pulse pressure, locomotor activity, and $\mathrm{HR}$ were very similar under $\mathrm{D} / \mathrm{D}$ and $\mathrm{L} / \mathrm{L}$ conditions, indicating that light, a principal entraining signal to the SCN, has little effect on impaired blood pressure circadian rhythm by smooth-muscle Bmal1 deletion.

To ensure that the observed blood pressure alterations in SMBmal1-KO mice resulted from smooth-muscle Bmal1 deletion, but not $\mathrm{Cre}$ recombinase expression, we measured blood pressure in the SM22 $\alpha$-Cre mice under 12:12 L/D conditions. No differences in either blood pressure level or circadian oscillations were observed between the age- and sex-matched SM22a-Cre mice and Bmal ${ }^{f l o x}$ ffox mice (Supplemental Figure 7A). muscle tone in SM-Bmal1-KO mice may have an effect on the baroreflex function, thus contributing to the loss of blood pressure circadian rhythm, we determined the spontaneous baroreflex sensitivity across the 24-hour day under 12:12 L/D conditions in conscious and free-moving SM-Bmal1-KO mice and WT littermates using the sequence method (31). Consistent with the previous reports in humans $(31,32)$, the spontaneous baroreflex sensitivity in WT littermates exhibited time-of-day variations with a higher sensitivity during the resting phase (the light phase in mice) than during the active phase (the dark phase in mice; Supplemental Figure 8). In contrast, the spontaneous baroreflex sensitivity in SM-Bmal1-KO mice was diminished across a 24-hour period, with a more pronounced decrease during the light phase. Importantly, the difference in spontaneous baroreflex sensitivity between light and dark phases was abolished in the SM-Bmal1-KO mice.

In summary, selective deletion of Bmal1 from smooth muscle but not from cardiomyocyte decreased the 24-hour MAP. In particular, deletion of Bmal1 from smooth muscle compromised blood pressure circadian rhythm without affecting locomotor activity. In addition, deletion of Bmal1 from smooth muscle altered 2 of the 3 


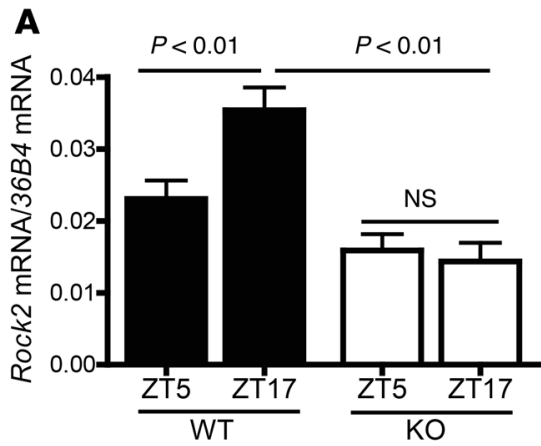

B

C

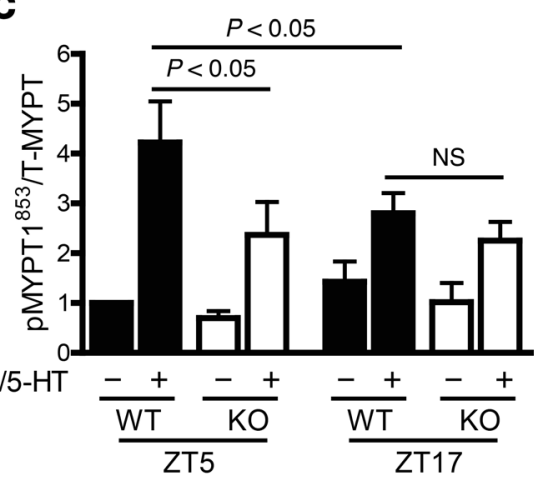

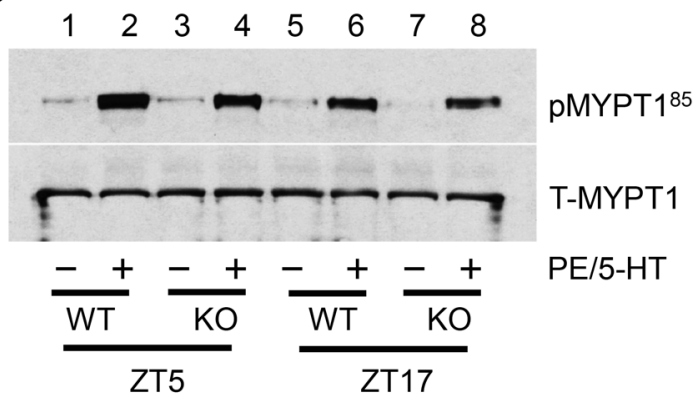

D

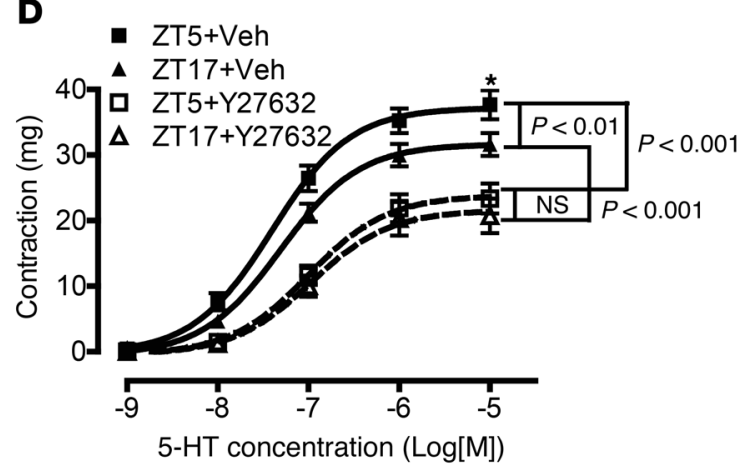

Figure 6. The time-of-day variation of Rock2 mRNA expression and ROCK2 kinase activity is regulated by BMAL1 and is involved in the time-of-day variation of smooth-muscle contraction.

(A) Real-time PCR analysis of Rock2 mRNA expression in mesenteric arteries $(n=3-5)$. (B and C) Representative Western blots (B) and quantitative data (C; $n=7-8)$ of total MYPT1 (T-MYPT1) and phosphorylated MYPT1 at Thr853 (p-MYPT1853) in mesenteric arteries. (D) Concentration-response curve showing that the time-of-day variation in vasoconstriction induced by 5 -HT was diminished by pretreatment of mesenteric arteries with Rho-kinase inhibitor Y27632 (10 $\mu \mathrm{M}, 30 \mathrm{~min}$; $n=7-8$ ). Veh, vehicle (DMSO). ${ }^{*} P<0.05$ vs. ZT17 + vehicle at $10 \mu \mathrm{M} 5-\mathrm{HT}$. major characteristics of the blood pressure oscillation: it decreased the oscillation amplitude and forward shifted the acrophase without affecting the period length. Moreover, deletion of Bmal1 from smooth muscle markedly elevated pulse pressure level, abolished pulse pressure circadian rhythm, and diminished the difference between the light and dark phases in baroreflex sensitivity.

Lack of Bmal1 in smooth muscle does not affect light conditioninduced change in blood pressure circadian oscillation. As shown in Figure 8A, in the WT littermates, the blood pressure oscillation amplitude gradually decreased from L/D to D/D and to L/L conditions. In contrast, the acrophase (Figure $8 \mathrm{~B}$ ) and period length (Figure $8 \mathrm{C}$ ) of the blood pressure circadian oscillation remained constant under $\mathrm{L} / \mathrm{D}$ and $\mathrm{D} / \mathrm{D}$ conditions, but significantly increased under L/L conditions. Interestingly, deletion of Bmal1 from smooth muscle significantly suppressed the blood pressure circadian oscillation amplitude and forward shifted the acrophase (Figure 7 and Supplemental Figures 5 and 6), but it had no detectable effect on L/L-induced changes in blood pressure circadian oscillations, including suppression in oscillation amplitude (Figure 8A), delay in acrophase (Figure $8 \mathrm{~B}$ ), and increase in oscillation period length (Figure $8 \mathrm{C}$ ) as well as L/L-induced changes in locomotor activity (Figure 8D). This indicates a minimal role of vascular smooth-muscle BMAL1 and contractility in light condition-induced alterations in blood pressure circadian oscillation.

\section{Discussion}

Major findings of the current study are as follows: (a) smooth-muscle-specific deletion of Bmal1 did not affect the central pacemaker SCN, but drastically suppressed the amplitude and the time-of-day variations in vasoconstriction in isolated preparations in response to various agonist stimulations, in myogenic response to pressure increase, and in in vivo pressor responses in anesthetized mice; (b) the inhibition of agonist-induced vasoconstriction was associated with suppression of $\mathrm{MLC}_{20}$ phosphorylation, Rock2 mRNA, and activity; moreover, BMAL1 directly bound to Rock2 promoter in a time-of-day-dependent manner in mesenteric arteries and was required for Rock2 promoter activity in cultured VSMCs; (c) in mice lacking smooth-muscle Bmal1, the blood pressure level was decreased; blood pressure circadian oscillation amplitude was decreased, whereas light-induced blood pressure changes were not affected; acrophase was forward shifted, but the period length and light-induced changes remained unaltered; and (d) smoothmuscle-specific deletion of Bmal1 markedly elevated pulse pressure level and abolished pulse pressure circadian rhythm.

How do our findings contribute to the current understanding of blood pressure circadian rhythm? It was long believed that all circadian rhythms, including blood pressure circadian rhythm, were primarily generated and controlled by the central pacemaker SCN. Indeed, ablation of the SCN results in the loss of circadian oscillation of blood pressure, along with the loss of endocrine and behavioral rhythms (33). However, recent studies illustrate that the behavioral circadian rhythm that is mainly controlled by the SCN does not correlate precisely with the blood pressure circadian rhythm in a 24 -hour period $(6,34)$, indicating an involvement of additional mechanisms in the generation and maintenance of blood pressure circadian rhythm. This concept is supported by the discovery that each of the core clock genes present in the SCN are also expressed and function in peripheral tissues (4-7). However, which clock gene in which peripheral tissue or tissues contributes to the blood pressure circadian rhythm remains elusive.

The results of the current study demonstrated that smoothmuscle BMAL1 substantially contributes to the maintenance of 
A
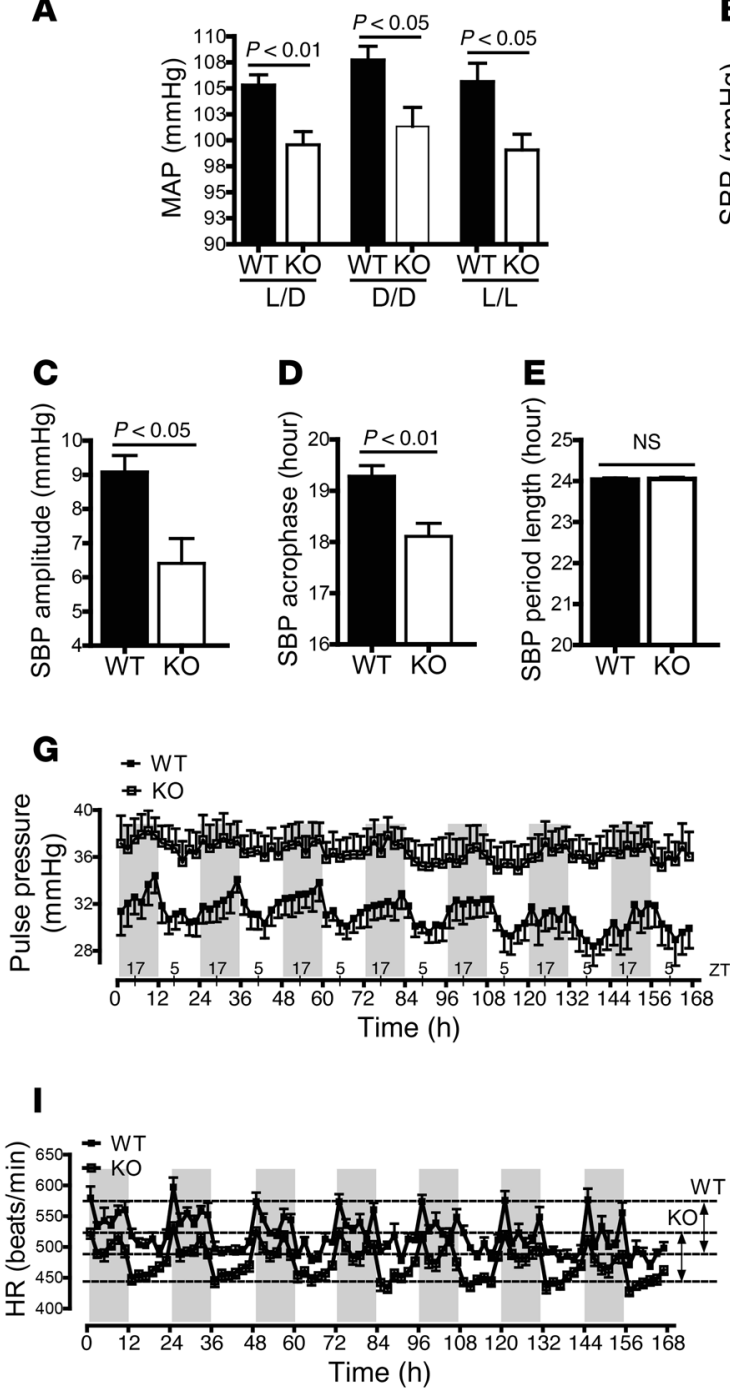

B

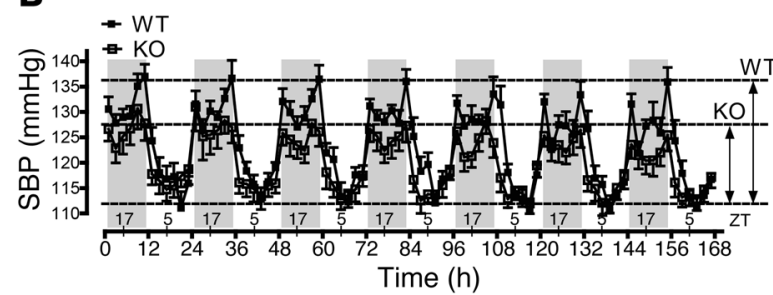

$\mathbf{F}$

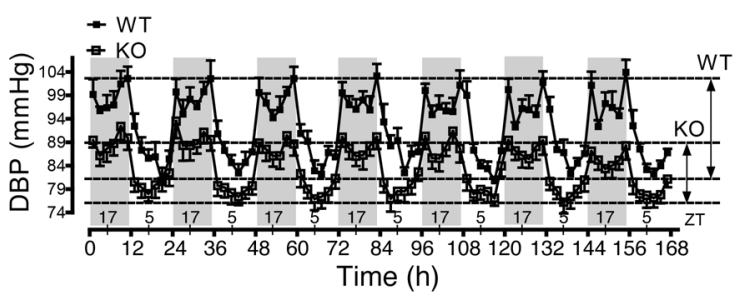

H

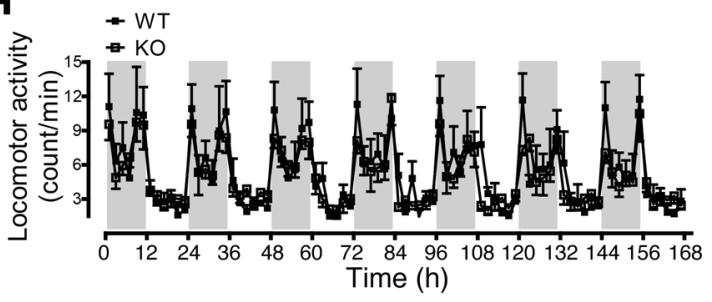

$\mathbf{J}$

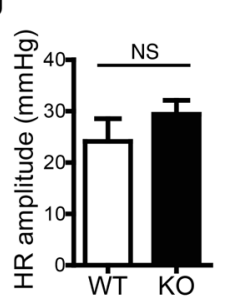

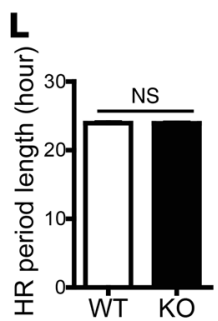

Figure 7. Circadian rhythms in blood pressure, but not in locomotor activity and HR, are altered in SM-Bmal1-KO mice under D/D conditions. (A) 24-hour MAP under 12:12 L/D, D/D, and L/L conditions $(n=5-8)$. (B and F-I) 7-day SBP (B), DBP (F), pulse pressure (C), locomotor activity (H), and HR (I) under D/D conditions $(n=5-7)$. (C-E) SBP circadian oscillations in amplitude (C), acrophase (D), and period length (E). (J-L) HR circadian oscillations in amplitude (J), acrophase (K), and period length (L).

normal blood pressure levels as well as blood pressure circadian rhythm. The MAP decreased by about 9 to $10 \mathrm{mmHg}$ in the global BMAL1-knockout mice (16). In mice lacking smooth-muscle Bmal1, an approximately $7 \mathrm{mmHg}$ blood pressure decrease was observed (Figure 7A), whereas in mice lacking cardiomyocyte Bmal1, no difference in blood pressure levels was observed (Supplemental Figure 7B), suggesting a major contribution of the smooth-muscle BMAL1 to the overall blood pressure level decrease in the global Bmal1-knockout mice. Moreover, blood pressure circadian rhythm amplitude and acrophase were significantly altered in mice lacking smooth-muscle Bmal1 under D/D (Figure 7, B-G), L/L (Supplemental Figure 5), and 12:12 L/D conditions (Supplemental Figure 6). A more striking effect of deleting smooth-muscle Bmal1 on blood pressure was observed in pulse pressure, with a dramatic increase in pulse pressure level and a complete loss of pulse pressure circadian rhythm (Figure 7G and Supplemental Figure 5G). While the significance of pulse pressure circadian rhythm remains elusive, multiple population-based cohort studies (35-37) and randomized trials of hypertension treatment (38) have shown that increased pulse pressure is associated with a variety of adverse cardiovascular outcomes, and there are reports that ambulatory monitoring of pulse pressure substantially refines the risk stratification in hypertensive patients $(39,40)$. Thus, our finding that smooth-muscle BMAL1 regulates the level and circadian rhythms of blood pressure including pulse pressure may have an important implication for human health.

How does smooth-muscle BMAL1 regulate blood pressure circadian rhythm? We and others have reported that vascular smooth-muscle contractile responses to various agonists exhibit a time-of-day variation (9-14). Therefore, one potential mechanism is that smooth-muscle BMAL1 regulates the time-of-day variation of vasoconstriction and thereby participates in the regulation of blood pressure circadian rhythm. Several lines of evidence from the current study support this potential mechanism. 
A

Strain: $P<0.001$

Treatment: $P<0.001$

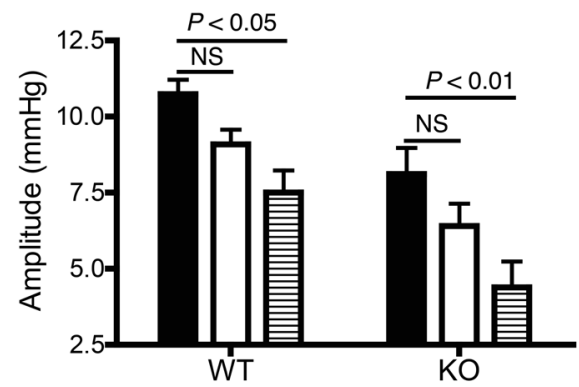

C

Strain: NS

Treatment: $P<0.001$
B
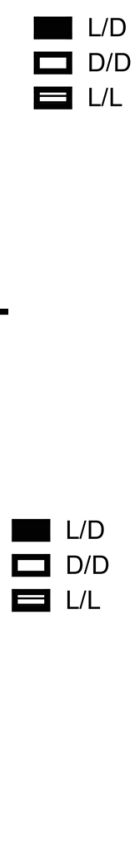

Strain: $P<0.001$

Treatment: $P<0.001$
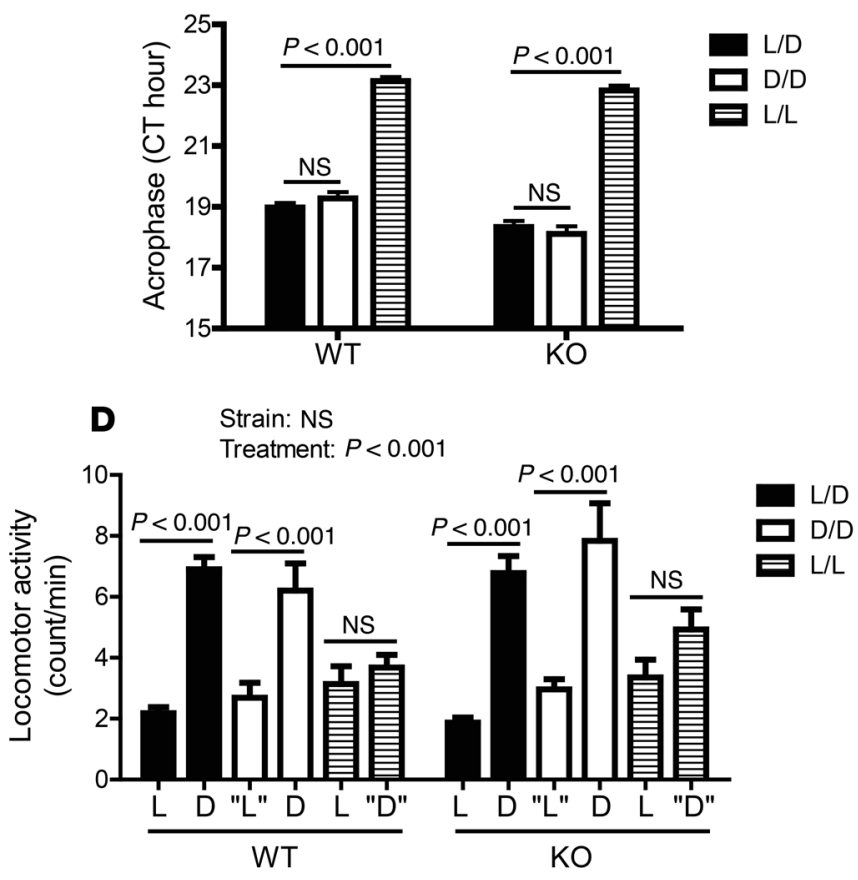

Figure 8. The change in blood pressure circadian rhythm with light is not altered in SM-Bmal1 mice. (A-C) The amplitude (A), acrophase (B), and period length $(C)$ of the rhythmicity were calculated from SBP data collected under $12: 12 \mathrm{~L} / \mathrm{D}, \mathrm{D} / \mathrm{D}$, and $\mathrm{L} / \mathrm{L}$ conditions $(n=8)$. (D) 12 -hour means of locomotor activity under $L / D, D / D$, and $L / L$ conditions $(n=8)$. "L", subjective light condition. " $D$ ", subjective dark condition.

First, the time-of-day variation in contractile responses to high $\mathrm{K}^{+}$depolarization and agonist (PE and 5-HT) stimulation was abolished in smooth-muscle Bmal1-deficient and endotheliumdenuded superior mesenteric arteries (Figure 2) and renal arteries (Supplemental Figure 2). We denuded endothelium, as we have usually done in our experiments $(9,41-44)$, to focus on studying smooth-muscle function, as a recent report demonstrated that selective deletion of a gene from smooth muscle affected endothelium function (45). Second, the myogenic responses were attenuated in the fifth branch of mesenteric arteries from SMBmal1-KO mice compared with that from WT littermates (Figure 3 , A and B). Surprisingly, a moderate inward eutrophic vascular remodeling was found in the fifth branch of mesenteric arteries (Supplemental Figure 3), but not in the second branch of mesenteric arteries (Supplemental Figure 1). Such selective and moderate vascular remodeling may be a direct effect of smooth-muscle Bmal1 deletion or a compensatory response to a decrease in blood pressure. Further studies are required to clarify this issue. Third, in line with these ex vivo vasoconstriction studies, the time-ofday variation in pressor response to $\mathrm{PE}$ was also diminished in anesthetized SM-Bmal1-KO mice (Figure 3C). Fourth, the timeof-day variation in $\mathrm{MLC}_{20}$ phosphorylation induced by agonist was attenuated in smooth-muscle Bmal1-deficient mesenteric arteries (Figure 4). However, one surprising finding of the current study is that the phase of vasoconstriction does not correlate with the phase of blood pressure level in WT littermates (Figure 2 vs. Figure 7, B, F, and G). This finding is unlikely to be attributable to the time delay caused by the ex vivo tissue preparation and tension measurement because we (Figure 3C) and others (46) have demonstrated a similar "antiphase" temporal relationship between the in vivo PE-induced pressor response and blood pressure. This paradoxical observation suggests the relationship between the time-of-day vasoconstriction variation and blood pressure circadian rhythm is complex rather than linear. This is not entirely unexpected, since vasoconstriction-induced blood pressure change will trigger sympathetic, endocrine, and local environmental changes via multiple mechanisms. Indeed, we (Supplemental Figure 8) and others $(31,32)$ have demonstrated that the spontaneous baroreflex sensitivity showed time-of-day variations, which is in phase with the time-of-day vasoconstriction variation (Supplemental Figure 8 vs. Figure 2).

The molecular mechanism underlying the time-of-day variation in vascular smooth-muscle contraction remained completely unknown until Saito et al. recently reported that, in cultured cells and isolated aorta, the expression and activity of ROCK2 exhibited a circadian rhythm in phase with that of MLC $_{20}$ phosphorylation (47). The current study demonstrates for the first time, to our knowledge, that, in isolated mesenteric arteries, BMAL1 directly binds to the E-box-containing region in the Rock2 promoter in a time-of-day-dependent manner (Figure 5, C and D), which is in phase with Rock2 mRNA expression (Figure 6A). A pivotal role of smooth-muscle BMAL1 in Rock2 transcriptional regulation was further demonstrated by complete loss of Rock2 promoter activity in Bmal1-deficient VSMCs and by the complete restoration of Rock2 promoter activity by restoring BMAL1 expression using adenovirus-mediated gene transfer (Figure 5E). Moreover, a temporal 
correlation of ROCK2 function (pMYPT ${ }^{853}$ phosphorylation) with vasoconstriction (Figure 6, B and C, vs. Figure 2) and $\mathrm{MLC}_{20}$ phosphorylation (Figure 6, B and C, vs. Figure 4) was observed in WT mice, but lost in SM-Bmal1-KO mice.

In summary, the current study provides several lines of evidence indicating that smooth-muscle BMAL1 is critical for timeof-day-dependent vasoconstriction and thereby blood pressure circadian rhythm. Moreover, the current study also reveals a mechanism by which smooth-muscle BMAL1 regulates MLC $_{20}$ phosphorylation and vasoconstriction via ROCK2 in a time-ofday-dependent manner. Since disruption of blood pressure circadian rhythm is implicated in many human diseases, including hypertension, acute myocardial infarction, sudden cardiac death, and stroke and is emerging as an index for future target organ damage and cardiovascular outcomes $(2,3)$, the new mechanistic insights into the daily control of vasoconstriction and blood pressure obtained in the current study could contribute to the foundation for future elucidation of the pathogenesis of many cardiovascular diseases involving disruption of blood pressure circadian rhythm.

\section{Methods}

Animals. SM-Bmal1-KO mice and iCS-Bmal1-KO mice were generated by crossing Bmal1 floxffox mice (18) with smooth-muscle-specific SM22 $\alpha$-Cre knockin mice (19) and cardiac-specific MerCreMer recombinase mice (30), respectively. SM-Bmal1-KO/Per2 ${ }^{\text {Luc }}$ mice were generated by crossing SM-Bmal1-KO mice with $m P e r 2^{\text {Luc }}$ knock-

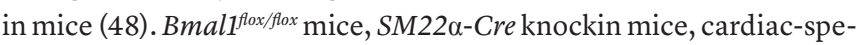
cific MerCreMer recombinase mice, and $m P e r 2^{L u c}$ knockin mice were purchased from the Jackson Laboratory. Only male mice at 12 to 16 weeks of age were used, and the mice were kept on a 12:12 L/D cycle unless indicated otherwise.

Analysis of smooth-muscle-specific Cre-mediated chromosome recombination in SM-Bmal1-KO mice. Genomic DNAs were extracted from various tissues of SM-Bmal1-KO mice and subjected to PCR analysis using a combination of 3 primers that were able to simultaneously detect both Bmal1-KO and WT (Bmal1 flox/flox) gene products as described (18).

Quantitative analysis of $m R N A$ expression. Mesenteric arteries were isolated from SM-Bmal1-KO mice and WT littermates at ZT5, ZT11, ZT17, and ZT23 (ZTO, light on; ZT12, light off). RNA extraction, cDNA synthesis, and real-time PCR were carried out as described (41, 49). The real-time PCR primers for mouse Per1 and Per2 were the same as those previously described (9). The real-time PCR primers for mouse Rock2 are described in Supplemental Table 2.

Real-time luminescence analysis of Per 2 gene expression. Mesenteric arteries and coronal brain sections containing SCN were isolated from SM-Bmal1-KO/Per $2^{\text {Luc }}$ mice and Per $2^{\text {Luc }}$ knockin mice at ZT10 to ZT11 and were then cultured in $35-\mathrm{mm}$ Petri dishes at $36^{\circ} \mathrm{C}$ in a light-tight, water-jacketed incubator. Luminescence was continuously recorded by LumiCycle (Actimetrics) as described (48).

Isometric tension measurement. Mesenteric arteries and right renal arteries were isolated from SM-Bmal1-KO mice and WT littermates at ZT5 or ZT17. Superior mesenteric arteries and renal arteries were cut into small spiral strips (about $3 \mathrm{~mm}$ in length and $350 \mu \mathrm{m}$ in width). Endothelium was denuded, and isometric contractions were measured as described $(9,41-44,50)$.
Assessment of mesenteric myogenic tone. The fifth branches of mesenteric arteries with an inner diameter of 55 to $85 \mu \mathrm{m}$ were prepared from SM-Bmal1-KO mice and WT littermates and then cannulated in a pressure myograph system (Living Systems Instrumentation). An active and passive pressure-diameter curve was recorded in the presence of $\mathrm{Ca}^{2+}$ and the absence of $\mathrm{Ca}^{2+}$ plus EGTA and nitroprusside, respectively, by $20 \mathrm{mmHg}$ stepwise increase of intraluminal pressure from 0 to $120 \mathrm{mmHg}$ in a physiological buffer at $37^{\circ} \mathrm{C}$ gassed with a $95 \% \mathrm{O}_{2}-5 \% \mathrm{CO}_{2}$ gas mixture as described (24).

Analysis of $M L C_{20}$ phosphorylation. Mesenteric arteries were isolated from SM-Bmal1-KO mice and WT littermates at ZT5 or ZT17. After equilibration in normal Krebs-Ringer bicarbonate buffer at $37^{\circ} \mathrm{C}$ for 30 minutes, mesenteric arteries were stimulated with 5-HT (10 $\mu \mathrm{M})$ plus PE $(100 \mu \mathrm{M})$ for 2 minutes, followed by immediate freezing in liquid nitrogen-chilled acetone containing $10 \%$ trichloroacetic acid. MLC $_{20}$ phosphorylation was then determined by urea/glycerolPAGE as described (24) and by immunoblots using a $\mathrm{MLC}_{20}$ phosphorylation-specific antibody (Thr18/Ser19; Cell Signaling Technology) as described $(49,51-53)$.

BMAL1 ChIP assay. A custom ChIP-grade BMAL1 rabbit polyclonal antibody was produced by Genemed Synthesis against mouse BMAL1 amino acids 59-66 (TDKDDPHGRLEYAEHQGR). BMAL1 antibody was purified from antisera by immunoaffinity chromatography using the antigen peptide coupled to agarose beads as described (49).

Mesenteric arteries were isolated from Bmal1 $1^{f 0 x / f f o x}$ mice at ZT5 and ZT17. Mesenteric arteries were fixed with $1 \%$ formaldehyde for 15 minutes to preserve the protein-DNA interactions. Mesenteric arteries were lysed in an SDS buffer containing protease inhibitors, and chromatin was fragmented by sonication. Three mesenteric arteries were pooled as 1 sample for BMAL1 ChIP analysis. The diluted chromatin was precleared by incubating with salmon sperm DNA (Life Technologies) and protein A/G agarose beads (Santa Cruz Biotechnology Inc.) and subjected to immunoprecipitation by incubating with the BMAL1 antibody $(2 \mu \mathrm{g})$ or an equal amount of nonspecific rabbit IgG (Vector Laboratory). The immune complexes were pulled down by protein A/G agarose beads and eluted. The crosslinks between protein and DNA were reversed by heating at $65^{\circ} \mathrm{C}$ for 4 hours. The released DNA was purified and amplified by PCR. The primers for mouse Per 1 , mouse Rock2 E-boxes 5 to 8, and mouse Rock2 E-boxes 9 to 10 are described in Supplemental Table 2.

Cloning mouse Rock2 promoter. A mouse bacterial artificial chromosome clone containing the mouse Rock2 promoter was purchased from Life Technologies. An approximately 3.2-kb PCR product $(-2,402$ to $+807 \mathrm{bp}$, relative to the translational initiation site) was amplified by PCR using primers as described in Supplemental Table 2, verified by DNA sequencing (Z. Guo and M. Gong, unpublished observations), and subcloned into the pGL3 basic vector (Promega) at the XhoI and KpnI sites.

ROCK2 promoter assay. Bmal1-deficient and WT VSMCs were isolated from SM-Bmal1-KO and WT littermate aortas as described (49, $54,55)$. Cells were cotransfected with pGl3-Rock2 luciferase vector and a Renilla luciferase using Lipofectamine-Plus Reagent (Life Technologies). A human BMAL1 adenovirus was purchased from Vector BioLabs and was purified by $\mathrm{CsCl}$ density gradient centrifugation as described (42). BMAL1 adenoviral expression in VSMC was verified by Western blot (Z. Guo and M. Gong, data not shown). Rock2 promoter activity was assayed by a modified dual luciferase enzyme assay as described (55). 
Telemetric measurement of blood pressure circadian rhythm and diurnal pressor responses. SM-Bmal1-KO mice and WT littermates were chronically instrumented in the left common carotid artery with a telemetry probe as described (27). After 10 days of recovery, SBP, DBP, MAP, pulse pressure, $\mathrm{HR}$, and locomotor activity data were collected for 3 consecutive days on a 12:12 L/D cycle, 7 consecutive days under $\mathrm{D} / \mathrm{D}$ conditions, and 7 consecutive days under $\mathrm{L} / \mathrm{L}$ conditions, respectively. Diurnal pressor responses to PE were measured in anesthetized mice either at ZT5 or ZT17 as described (9).

Statistics. All data were expressed as mean \pm SEM. For comparison of 1 parameter between 2 strains of mice, statistical analysis was performed using 2-tailed, unpaired Student's $t$ test. For comparison of multiple parameters between 2 strains of mice at a single time point, statistical analysis was performed using 1-way ANOVA with a Newman-Keuls post test. For comparison of multiple parameters between 2 strains of mice across various ZT time points, various concentrations, or various pressures, statistical analysis was performed using 2-way ANOVA with repeated measures and Bonferroni's post test. $P<0.05$ was considered significant. $P \geq 0.05$ was considered NS.
Study approval. All animal procedures were approved by the Institutional Animal Care and Use Committee of the University of Kentucky.

\section{Acknowledgments}

This work was supported by NIH grants HL088389 (to Z. Guo), HL106843 (to M.C. Gong and Z. Guo), and RC1ES018636 (to K. Esser and M.C. Gong) as well as a National Institute of General Medical Sciences grant (P20 GM103527-05 to L. Cassis). We thank Ming Zhang for her excellent technical assistance in breeding mice.

Address correspondence to: Ming C. Gong, Department of Physiology, College of Medicine, University of Kentucky, 900 South Limestone, 509 Wethington Building, Lexington, Kentucky 40536, USA. Phone: 859.218.1361; E-mail: ming.gong@uky.edu. Or to: Zhenheng Guo, Department of Internal Medicine, College of Medicine, University of Kentucky, 900 South Limestone, 515 Wethington Building, Lexington, Kentucky, USA. Phone: 859.218.1416;E-mail: zguo2@uky.edu.
1. Millar-Craig MW, Bishop CN, Raftery EB. Circadian variation of blood-pressure. Lancet. 1978;1(8068):795-797.

2. Cohen MC, Rohtla KM, Lavery CE, Muller JE, Mittleman MA. Meta-analysis of the morning excess of acute myocardial infarction and sudden cardiac death. Am J Cardiol. 1997;79(11):1512-1516.

3. Elliott WJ. Circadian variation in the timing of stroke onset: a meta-analysis. Stroke. 1998;29(5):992-996.

4. Reilly DF, Westgate EJ, FitzGerald GA. Peripheral circadian clocks in the vasculature. Arterioscler Thromb Vasc Biol. 2007;27(8):1694-1705.

5. Lowrey PL, Takahashi JS. Mammalian circadian biology: elucidating genome-wide levels of temporal organization. Annu Rev Genomics Hum Genet. 2004;5:407-441.

6. Jones H, Atkinson G, Leary A, George K, Murphy $\mathrm{M}$, Waterhouse J. Reactivity of ambulatory blood pressure to physical activity varies with time of day. Hypertension. 2006;47(4):778-784.

7. Zylka MJ, Shearman LP, Weaver DR, Reppert SM. Three period homologs in mammals: differential light responses in the suprachiasmatic circadian clock and oscillating transcripts outside of brain. Neuron. 1998;20(6):1103-1110.

8. Somlyo AP, Somlyo AV. Signal transduction and regulation in smooth muscle. Nature. 1994;372(6503):231-236.

9. Su W, Xie Z, Guo Z, Duncan MJ, Lutshumba J, Gong MC. Altered clock gene expression and vascular smooth muscle diurnal contractile variations in type 2 diabetic $\mathrm{db} / \mathrm{db}$ mice. Am JPhysiol Heart Circ Physiol. 2012;302(3):H621-H633.

10. Gohar M, Daleau P, Atkinson J, Gargouil YM. Ultradian variations in sensitivity of rat aorta rings to noradrenaline. Eur J Pharmacol. 1992;229(1):69-73.

11. Witte K, Hasenberg T, Rueff T, Hauptfleisch S, Schilling L, Lemmer B. Day-night variation in the in vitro contractility of aorta and mesenteric and renal arteries in transgenic hypertensive rats.
Chronobiol Int. 2001;18(4):665-681.

12. Gorgun CZ, Keskil ZA, Hodoglugil U, Ercan ZS, Abacioglu N, Zengil H. In vitro evidence of tissue susceptibility rhythms. I. Temporal variation in effect of potassium chloride and phenylephrine on rat aorta. Chronobiol Int. 1998;15(1):39-48.

13. Keskil Z, Gorgun CZ, Hodoglugil U, Zengil H. Twenty-four-hour variations in the sensitivity of rat aorta to vasoactive agents. Chronobiol Int. 1996;13(6):465-475.

14. Andreotti F, et al. Circadianicity of hemostatic function and coronary vasomotion. Cardiologia. 1999;44 suppl 1(pt 1):245-249.

15. Bunger MK, et al. Progressive arthropathy in mice with a targeted disruption of the Mop3/ Bmal-1 locus. Genesis. 2005;41(3):122-132.

16. Curtis AM, Cheng Y, Kapoor S, Reilly D, Price TS, Fitzgerald GA. Circadian variation of blood pressure and the vascular response to asynchronous stress. Proc Natl Acad Sci US A. 2007;104(9):3450-3455.

17. McDearmon EL, et al. Dissecting the functions of the mammalian clock protein BMAL1 by tissue-specific rescue in mice. Science. 2006;314(5803):1304-1308.

18. Storch KF, et al. Intrinsic circadian clock of the mammalian retina: importance for retinal processing of visual information. Cell. 2007;130(4):730-741.

19. Zhang J, et al. Generation of an adult smooth muscle cell-targeted Cre recombinase mouse model. Arterioscler Thromb Vasc Biol. 2006;26(3):e23-e24.

20. Chang L, et al. Vascular smooth muscle cell-selective peroxisome proliferator-activated receptor- $\gamma$ deletion leads to hypotension. Circulation. 2009;119(16):2161-2169.

21. Rudic RD, et al. BMAL1 and CLOCK, two essential components of the circadian clock, are involved in glucose homeostasis. PLoS Biol. 2004;2(11):e377.

22. Kondratov RV, Kondratova AA, Gorbacheva
VY, Vykhovanets OV, Antoch MP. Early aging and age-related pathologies in mice deficient in BMAL1, the core componentof the circadian clock. Genes Dev. 2006;20(14):1868-1873.

23. McCurley A, et al. Direct regulation of blood pressure by smooth muscle cell mineralocorticoid receptors. Nat Med. 2012;18(9):1429-1433.

24. Su W, Xie Z, Liu S, Calderon LE, Guo Z, Gong MC. Smooth muscle-selective CPI-17 expression increases vascular smooth muscle contraction and blood pressure. Am JPhysiol Heart Circ Physiol. 2013;305(1):H104-H113.

25. Shimokawa H, Takeshita A. Rho-kinase is an important therapeutic target in cardiovascular medicine. Arterioscler Thromb Vasc Biol. 2005;25(9):1767-1775.

26. Golombek DA, Rosenstein RE. Physiology of circadian entrainment. Physiol Rev. 2010;90(3):1063-1102.

27. Su W, Guo Z, Randall DC, Cassis L, Brown DR, Gong MC. Hypertension and disrupted blood pressure circadian rhythm in Type 2 diabetic $\mathrm{db} / \mathrm{db}$ mice. Am J Physiol Heart Circ Physiol. 2008;295(4):H1634-H1641.

28. Hughes ME, Hogenesch JB, Kornacker K. JTK CYCLE: an efficient nonparametric algorithm for detecting rhythmic components in genome-scale data sets. J Biol Rhythms. 2010;25(5):372-380.

29. Sohal DS, et al. Temporally regulated and tissuespecific gene manipulations in the adult and embryonic heart using a tamoxifen-inducible Cre protein. Circ Res. 2001;89(1):20-25.

30. Schroder EA, et al. The cardiomyocyte molecular clock, regulation of Scn5a, and arrhythmia susceptibility. Am J Physiol Cell Physiol. 2013;304(10):C954-C965.

31. Di Rienzo M, Parati G, Castiglioni P, Tordi R, Mancia G, Pedotti A. Baroreflex effectiveness index: an additional measure of baroreflex control of heart rate in daily life. Am J Physiol Regul Integr Comp Physiol. 2001;280(3):R744-R751.

32. Hossmann V, Fitzgerald GA, Dollery CT. Cir- 
cadian rhythm of baroreflex reactivity and adrenergic vascular response. Cardiovasc Res. 1980;14(3):125-129.

33. Witte K, et al. Effects of SCN lesions on circadian blood pressure rhythm in normotensive and transgenic hypertensive rats. Chronobiol Int. 1998;15(2):135-145.

34. Ivanov P, Hu K, Hilton MF, Shea SA, Stanley HE. Endogenous circadian rhythm in human motor activity uncoupled from circadian influences on cardiac dynamics. Proc Natl Acad Sci U S A. 2007;104(52):20702-20707.

35. Thomas F, Blacher J, Benetos A, Safar ME, Pannier $B$. Cardiovascular risk as defined in the 2003 European blood pressure classification: the assessment of an additional predictive value of pulse pressure on mortality. JHypertens. 2008;26(6):1072-1077.

36. Domanski M, et al. Pulse pressure and cardiovascular disease-related mortality: follow-up study of the Multiple Risk Factor Intervention Trial (MRFIT). JAMA. 2002;287(20):2677-2683.

37. Lorenzo C, Aung K, Stern MP, Haffner SM. Pulse pressure, prehypertension, and mortality: the San Antonio heart study. Am J Hypertens. 2009;22(11):1219-1226.

38. Vaccarino V, et al. Pulse pressure and risk of cardiovascular events in the systolic hypertension in the elderly program. Am J Cardiol. 2001;88(9):980-986.

39. Verdecchia P, Schillaci G, Borgioni C, Ciucci A, Pede S, Porcellati C. Ambulatory pulse pressure: a potent predictor of total cardiovascular risk in hypertension. Hypertension. 1998;32(6):983-988.

40. Staessen JA, et al. Ambulatory pulse pressure as predictor of outcome in older patients with systolic hypertension. Am J Hypertens. 2002; 15(10 pt 1):835-843.

41. Guo Z, et al. COX-2 up-regulation and vascular smooth muscle contractile hyperreactivity in spontaneous diabetic $\mathrm{db} / \mathrm{db}$ mice. Cardiovasc Res. 2005;67(4):723-735.

42. Guo Z, Su W, Ma Z, Smith GM, Gong MC. Ca ${ }^{2+}$ independent phospholipase $\mathrm{A} 2$ is required for agonist-induced $\mathrm{Ca}^{2+}$ sensitization of contraction in vascular smooth muscle. J Biol Chem. 2003;278(3):1856-1863.

43. Su W, Xie Z, Guo Z, Duncan MJ, Lutshumba J, Gong MC. Altered clock gene expression and vascular smooth muscle diurnal contractile variations in type 2 diabetic $\mathrm{db} / \mathrm{db}$ mice. Am J Physiol Heart Circ Physiol. 2011;302(3):H621-H633.

44. Xie Z, Gong MC, Su W, Xie D, Turk J, Guo Z. Role of calcium-independent phospholipase A2 $\beta$ in high glucose-induced activation of RhoA, Rho kinase, and CPI-17 in cultured vascular smooth muscle cells and vascular smooth muscle hypercontractility in diabetic animals. J Biol Chem. 2010;285(12):8628-8638

45. Pelham CJ, et al. Cullin-3 regulates vascular smooth muscle function and arterial blood pressure via PPAR $\gamma$ and RhoA/Rho-kinase. Cell Metab. 2012;16(4):462-472.

46. Masuki S, Todo T, Nakano Y, Okamura H, Nose H. Reduced alpha-adrenoceptor responsiveness and enhanced baroreflex sensitivity in Cry-deficient mice lacking a biological clock. J Physiol. 2005;566(pt 1):213-224.

47. Saito T, et al. Pivotal role of Rho-associated kinase 2 in generating the intrinsic circadian rhythm of vascular contractility. Circulation. 2013;127(1):104-114.

48. Yoo SH, et al. PERIOD2::LUCIFERASE realtime reporting of circadian dynamics reveals persistent circadian oscillations in mouse peripheral tissues. Proc Natl Acad Sci U S A. 2004;101(15):5339-5346.

49. Xie Z, Gong MC, Su W, Turk J, Guo Z. Group
VIA phospholipase A2 (iPLA2beta) participates in angiotensin II-induced transcriptional upregulation of regulator of g-protein signaling-2 in vascular smooth muscle cells. J Biol Chem. 2007;282(35):25278-25289.

50. Gong MC, et al. Myosin light chain phosphatase activities and the effects of phosphatase inhibitors in tonic and phasic smooth muscle. J Biol Chem. 1992;267(21):14662-14668.

51. Xie Z, Su W, Guo Z, Pang H, Post SR, Gong MC. Up-regulation of CPI-17 phosphorylation in diabetic vasculature and high glucose cultured vascular smooth muscle cells. Cardiovasc Res. 2006;69(2):491-501.

52. Pang H, Guo Z, Su W, Xie Z, Eto M, Gong MC. RhoA-Rho kinase pathway mediates thrombinand U-46619-induced phosphorylation of a myosin phosphatase inhibitor, CPI-17, in vascular smooth muscle cells. Am J Physiol Cell Physiol. 2005;289(2):C352-C360.

53. Pang H, Guo Z, Xie Z, Su W, Gong MC. Divergent kinase signaling mediates agonistinduced phosphorylation of phosphatase inhibitory proteins PHI-1 and CPI-17 in vascular smooth muscle cells. Am J Physiol Cell Physiol. 2006;290(3):C892-C899.

54. Xie Z, Gong MC, Su W, Xie D, Turk J, Guo Z. Role of calcium-independent phospholipase A2beta in high glucose-induced activation of RhoA, Rho kinase, and CPI-17 in cultured vascular smooth muscle cells and vascular smooth muscle hypercontractility in diabetic animals. J Biol Chem. 2010;285(12):8628-8638.

55. Xie Z, et al. Identification of a cAMP-response element in the regulator of G-protein signaling-2 (RGS2) promoter as a key cis-regulatory element for RGS2 transcriptional regulation by angiotensin II in cultured vascular smooth muscles. J Biol Chem. 2011;286(52):44646-44658. 